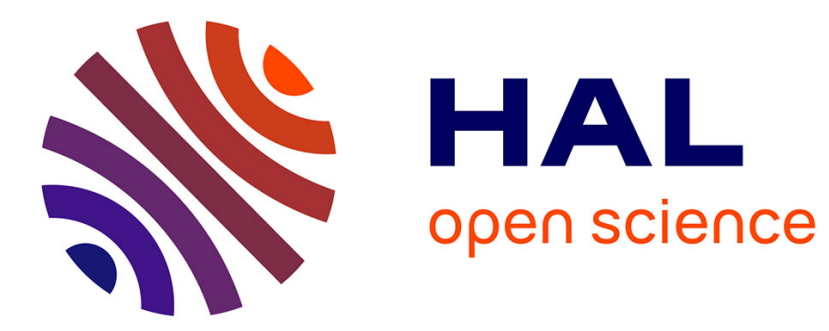

\title{
Adaptive Terminal Sliding Mode Control of a Redundantly-Actuated Cable-Driven Parallel Manipulator: CoGiRo
}

Gamal El-Ghazaly, Marc Gouttefarde, Vincent Creuze

\section{- To cite this version:}

Gamal El-Ghazaly, Marc Gouttefarde, Vincent Creuze. Adaptive Terminal Sliding Mode Control of a Redundantly-Actuated Cable-Driven Parallel Manipulator: CoGiRo. CableCon: Cable-Driven Parallel Robots, Tobias Bruckmann; Andreas Pott, Aug 2014, Duisburg, Germany. pp.179-200, 10.1007/978-3-319-09489-2_13. lirmm-01084648

\section{HAL Id: lirmm-01084648 \\ https://hal-lirmm.ccsd.cnrs.fr/lirmm-01084648}

Submitted on 19 Nov 2014

HAL is a multi-disciplinary open access archive for the deposit and dissemination of scientific research documents, whether they are published or not. The documents may come from teaching and research institutions in France or abroad, or from public or private research centers.
L'archive ouverte pluridisciplinaire HAL, est destinée au dépôt et à la diffusion de documents scientifiques de niveau recherche, publiés ou non, émanant des établissements d'enseignement et de recherche français ou étrangers, des laboratoires publics ou privés. 


\title{
Adaptive terminal sliding mode control of a redundantly-actuated cable-driven parallel manipulator: CoGiRo
}

Gamal El-Ghazaly, Marc Gouttefarde, and Vincent Creuze

\begin{abstract}
This paper presents an extended adaptive control scheme via terminal sliding mode (TSM) for cable-driven parallel manipulators (CDPM). Compared with linear hyperplane-based sliding mode control, TSM is able to guarantee highprecision and robust tracking performances which arise from its main feature of finite-time convergence. This motivates applying TSM to robotic manipulators in general and, as presented in this paper, to CDPM in particular. The scheme presented in this paper extends early developed TSM control schemes which are based on partial knowledge of system dynamics. Instead, making use of the property that the dynamic models of mechanical manipulators are linear in inertial parameters, an adaptive control law is synthesised based on an appropriate choice of Lyapunov function which guarantees finite-time convergence to neighborhood of sliding mode. A key challenge of the control of CDPM is that cable tensions must be admissible, i.e. lying in a non-negative range of admissible values. As long as cable tensions are admissible, the overall dynamics of CDPM can be easily written in either actuator space or operational space which in turn facilitates control system design. The extended adaptive control scheme has been applied to a large redundantly actuated CDPR prototype, CoGiRo. Simulation results show the effectiveness of the proposed control method.
\end{abstract}

\section{Introduction}

Cable-driven parallel manipulator (CDPM) is a particular type of robots in which the motion of the platform (end-effector) is provided by varying the length of the cables. Compared with classical parallel manipulators which are characterised by their

Gamal El-Ghazaly, Marc Gouttefarde, and Vincent Creuze

Laboratoire d'Informatique, de Robotique et de Microélectronique de Montpellier (LIRMM, CNRS-UM2), 161 Rue Ada, 34392, Montpellier, France e-mail: \{gamal.elghazaly, marc.gouttefarde, vincent.creuze\}@lirmm.fr 
limited workspace, CDPMs can have a very large workspace and also capable of manipulating heavy loads at high operating speed [1] which make them useful characteristics for many potential industrial applications. For crane-like applications [2] such as pick and place tasks [3], suspended CDPM are usually used as they reduce the risk of cable collisions in the workspace area. Cable-suspended CDPM are not fully-constrained and cannot have wrench closure over the entire workspace. However, a suspended CDPM uses the mobile platform and payload weights to keep the cables in tension. Furthermore, using more cables than DOFs in cable-suspended CDPM can allow the robot to have a significantly wider workspace $[3,4,5]$. In this paper, a 6-DOF suspended CDPM prototype with two degrees of actuation redundancy is used to evaluate the performance of the proposed TSM control scheme.

Many challenges arise in CDPMs among which control design is a difficult one. These challenges stem from the fact that cables can only apply tension forces imposing a strict constraint on control inputs. For redundantly actuated CDPM, an infinite number of cable tension distributions exist for a given external forces applied to the platform. The redundancy resolution has attracted the attention of many researchers. The proposed algorithms to resolve redundancy can be categorised into two main classes namely, iterative algorithms and non-iterative algorithms. Iterative algorithms are usually based on optimization and are not always suitable for real time implementations $[6,7,8]$. On the other hand, non-iterative algorithms give a solution in a reasonable amount of time and can be more easily implemented in real-time $[9,10,5]$.

Compared to the great deal of attention that has been paid to motion control of rigid-link manipulators, only relatively few results of control of CDPM exist in the literature. Gholami et-al. [11] proposed an operational-space PD controller for trajectory tracking. Kawamura et-al. proposed a PD controller with gravity compensation in [1]. The PD controller is designed in the actuator-space with Lyapunov stability. A robust Lyapunov-based design of PID tracking controller has been introduced for a fully-constrained planar CDPM in [12]. A PD controller with adaptive compensation has been applied to fully-constrained CDPM in [13]. Computedtorque control methods appeared in [14]. To make use of the advantages of both the actuator-space and operational-space formulations, a dual space adaptive controller has been proposed in [15].

Sliding mode control (SMC) has been found effective to deal with dynamic systems with uncertainties, time-varying parameters, and bounded external disturbances [16]. The main idea of SMC is to force the states of the system to stay in a chosen switching manifold satisfying a desired dynamic behavior. The choice of linear hyperplanes as switching manifolds guarantee asymptotic stability i.e. the closed-loop error converges to the neighborhood of the origin as the time approachs to infinity. Although classical SMC gave reasonable performance for robotic manipulators, only few results for CDPMs exist in the literature [17]. The performance of classical SMC could be enhanced if the closed-loop errors are forced to reach the origin in finite time. SMC with finite-time stabilization are called terminal sliding mode (TSM). Several ideas of designing TSM based control schemes have been developed to achieve finite-time stabilization in [18, 19, 20, 21]. The control schemes 
based on those ideas are able to guarantee high-precision and robust tracking performances due to finite-time convergence even with high uncertainty. This property justifies adopting TSM based control schemes for robotic manipulators and motivates us to use it for CDPMs. TSM control schemes developed for rigid-link manipulators may not exploit their properties very well. Even adaptive TSM control schemes developed for rigid-link manipulators do not rely on the linearity of inertial parameters [22]. In [18], a robust TSM control has been developed for robotics manipulators. However, the main problem of this scheme is that controller singularity may be reached in sliding mode. A non-singular TSM has been proposed for robotic manipulators in [19] where a new terminal sliding manifold has been proposed to avoid controller singularity. However, this control scheme did not eliminate chattering in control inputs. A continuous and non-singular TSM control scheme has been proposed also for robotic manipulators in [20] to avoid both chattering and singularity. However, the proposed schemes in $[18,19,20]$ did not consider the aforementioned property of the dynamic model of robotics manipulator and therefore cannot be directly applied to control CDPM for which high uncertainty may appear specially when manipulating payloads of varying sizes and weights. Instead, adaptive TSM control scheme has been proposed also for robotic manipulators in [23] where the Coriolis, centrifugal and gravity terms are approximated by polynomials of first order. The role of the adaptive mechanism is to estimate the coefficients of these polynomials which approximate the manipulator dynamics. However, [23] did not estimate the true inertial parameters of the manipulator which may give relatively large control inputs specially when loads are introduced.

This paper extends an early developed non-singular continuous TSM control scheme for robotic manipulators proposed in [20] by incorporating an adaptive mechanism to estimate dynamic parameters and by applying it to CDPM. The scheme in [20] is based on the assumption that the nominal values of inertial matrix components as well as of Coriolis, centrifugal and gravity forces are known and their corresponding uncertainties have known bounds. It is worth noting that the CDPMs considered in this paper are designed to carry heavy payloads having different weights so that the mobile platform inertial parameters are subjected to large variations. Therefore, if the scheme in [20] is directly applied, very high control input torques may be required especially during loading situations. The most appropriate solution to this problem is to estimate online the dynamic parameters of the CDPM via an adaptive mechanism. In this paper, the overall dynamics of CDPMs (both winches and platform) is formulated to be linear in all inertial and friction parameters. Thereby, it facilitates the design of the adaptive control scheme. The whole control scheme is synthesised and analysed based on Lyapunov stability theory. Moreover, it guarantees the finite-time convergence of the closed-loop system. To show its effectiveness, the proposed control scheme is applied to a large redundantly actuated CDPM, CoGiRo [3].

The paper is organised as follows. In Section 2, kinematic and dynamic modeling of CDPMs are presented and some properties of the dynamic model are recalled. Section 3 focuses on the proposed adaptive terminal sliding mode control scheme where finite-time convergence stability of the overall closed-loop system is analysed 
via Lyapunov direct method. Finally, to show the effectiveness of the proposed control scheme, a simulation study for pick-and-place task is performed on the CoGiRo CDPM in Section 4. Section 5 concludes this paper.

\section{Kinematic and Dynamic Modeling of CDPM}

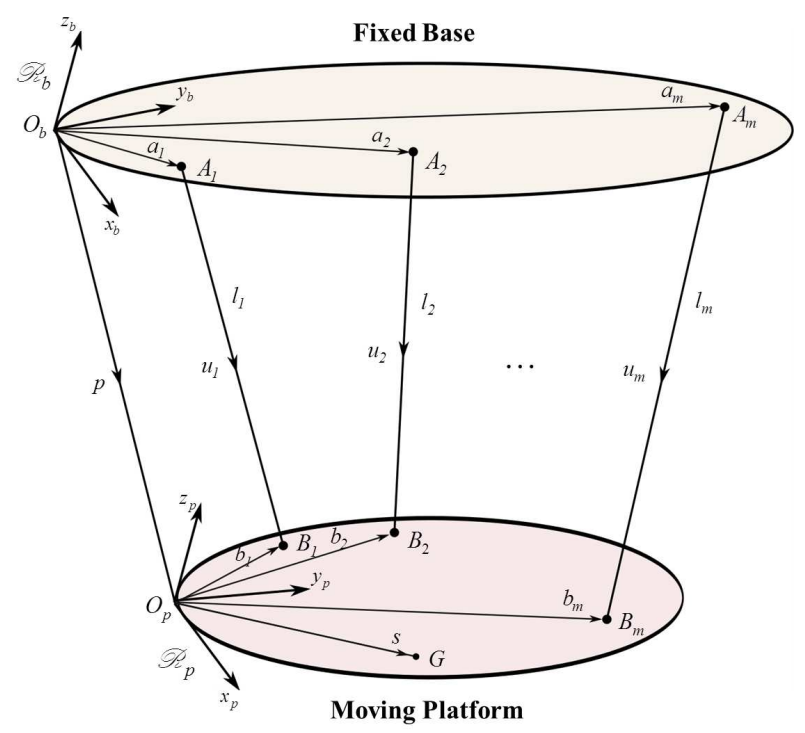

Fig. 1: Schematic Diagram of a General CDPM

In this section, kinematic and dynamic modeling of CDPM are presented. We assume that the elasticity of cables is negligible and cables almost behave as massless rigid strings. Modeling of CDPM has been presented in many research work before e.g. $[24,25,26,3]$. However, in this section, our main objective is to present the overall dynamics of CDPM (both winches and platform) in compact form and linear in all dynamic parameters. This compact form facilitates the design of adaptive control schemes in general $[22,27]$ and is particularly suitable for the adaptive control scheme introduced in this paper. The notations of a general CDPM for kinematic modeling is shown in Fig. 1. 


\subsection{Kinematic Model}

As shown in Fig. 1, CDPM is composed of a moving platform linked to a fixed base through cables with varying lengths via winches which are generally attached to the base structure. Let $\mathscr{R}_{b}$ and $\mathscr{R}_{p}$ denote two frames assigned to the base and moving platform, respectively. In the case of CDPM with $m$ cables, let cable $i$ connects the point $A_{i}$ at which it extends from the base frame to point $B_{i}$ at which it is attached to the moving platform. The position and orientation $\mathscr{R}_{p}$ with respect to $\mathscr{R}_{b}$ is defined by the configuration vector $\mathbf{x}=\left[p^{T} \varphi^{T}\right]^{T}$ where $p=[x y z]^{T}$ is the position vector and $\varphi=\left[\begin{array}{lll}\phi & \theta\end{array}\right]^{T}$ is a vector representing the orientation of $\mathscr{R}_{p}$ with respect to $\mathscr{R}_{b}$ using Euler angles. The rotation matrix from $\mathscr{R}_{b}$ to $\mathscr{R}_{p}$ denoted by $\mathbf{R}_{b}^{p}$ is represented by three successive rotations as

$$
\mathbf{R}_{b}^{p}(\phi, \theta, \psi)=\boldsymbol{\operatorname { R o t }}(x, \phi) \boldsymbol{\operatorname { R o t }}(y, \theta) \boldsymbol{\operatorname { R o t }}(z, \psi) .
$$

Using the definition of elementary rotations around coordinate axes, $\mathbf{R}_{b}^{p}$ can be written as

$$
\mathbf{R}_{b}^{p}(\phi, \theta, \psi)=\left[\begin{array}{ccc}
C \theta C \psi & -C \theta S \psi & S \theta \\
C \phi S \psi+S \phi S \theta C \psi & C \phi C \psi-S \phi S \theta S \psi & -S \phi C \theta \\
S \phi S \psi-C \phi S \theta C \psi S \phi C \psi+C \phi S \theta S \psi & C \phi C \theta
\end{array}\right]
$$

where $C(\cdot)=\cos (\cdot)$ and $S(\cdot)=\sin (\cdot)$. If $q_{i}$ denotes motor $i$ angular position and $r_{i}$ represents motor $i$ angular position to cable length transmission ratio, then the cable length $l_{i}$ can be expressed as

$$
l_{i}=r_{i} q_{i}
$$

Let $\mathbf{q}=\left[\begin{array}{llll}q_{1} & q_{2} & \ldots & q_{m}\end{array}\right]^{T} \in \mathbb{R}^{m}$, and $\mathbf{L}=\left[\begin{array}{llll}l_{1} & l_{2} & \ldots & l_{m}\end{array}\right]^{T} \in \mathbb{R}^{m}$ be two vectors gathering angular position variables and cable lengths, respectively. Then, one can write (3) in a vector matrix form as

$$
\mathbf{L}=\mathbf{R q},
$$

where $\mathbf{R}$ is a diagonal matrix containing the transmission ratios $r_{i}$ i.e.

$$
\mathbf{R}=\operatorname{diag}\left\{r_{1}, r_{2}, \ldots, r_{m}\right\}
$$

Let $a_{i}$ denotes the position vector corresponding to point $A_{i}$ expressed in $\mathscr{R}_{b}, b_{i}$ denotes the position vector corresponding to point $B_{i}$ expressed in $\mathscr{R}_{p}$, and $u_{i}$ is a vector along the direction of cable $i$ and has the same magnitude of the cable length. Given the position and orientation of the moving platform $\mathbf{x}$, the loop closure of cable $i$ expressed in $\mathscr{R}_{b}$ can be written as follows

$$
u_{i}=p-a_{i}+\mathbf{R}_{b}^{p} b_{i}
$$

The length of cable $i$ is then computed as

$$
l_{i}^{2}=u_{i}^{T} u_{i}=\left[p+\mathbf{R}_{b}^{p} b_{i}-a_{i}\right]^{T}\left[p+\mathbf{R}_{b}^{p} b_{i}-a_{i}\right] .
$$


Differentiating (7) with respect to time together with (4) gives

$$
\dot{\mathbf{L}}=\mathbf{R} \dot{\mathbf{q}}=\mathbf{J}(\mathbf{x}) \dot{\mathbf{x}},
$$

where $\mathbf{J}(\mathbf{x})$ defines a $6 \times m$ Jacobian matrix of the CDPM. The second-order derivative (7) with respect is then written as

$$
\ddot{\mathbf{L}}=\mathbf{R} \ddot{\mathbf{q}}=\mathbf{J}(\mathbf{x}) \ddot{\mathbf{x}}+\dot{\mathbf{J}}(\mathbf{x}, \dot{\mathbf{x}}) \dot{\mathbf{x}}
$$

\subsection{Platform Dynamics}

Let $\mathbf{S}=\left[\begin{array}{lll}\mathrm{S}_{x} & \mathrm{~S}_{y} & \mathrm{~S}_{z}\end{array}\right]^{\mathrm{T}}$ be a position vector of the platform COM $G$ in $\mathscr{R}_{p}$. Let $\mathbf{I}_{p}=$ $\mathbf{I}_{o}-\mathbf{M} \hat{\mathbf{S}} \hat{\mathbf{S}}$ be the inertia tensor of the platform with respect to the origin of $\mathscr{R}_{p}$ and expressed in the same frame, where $\mathbf{I}_{o}$ is the inertia tensor with respect to COM of the platform, $\mathbf{M}$ is the total platform mass, and $\hat{\mathbf{S}}$ denotes the $3 \times 3$ skew-symmetric matrix associated to $\mathbf{S}$. Let us denote the matrix $\mathbf{I}_{p}$ as

$$
\mathbf{I}_{p}=\left[\begin{array}{llll}
\mathrm{XX} & \mathrm{XY} & \mathrm{XZ} \\
\mathrm{XY} & \mathrm{YY} & \mathrm{YZ} \\
\mathrm{XZ} & \mathrm{YZ} & \mathrm{ZZ}
\end{array}\right] .
$$

Let MS be a vector representing the first moment of the moving platform which can be expressed as

$$
\mathbf{M S}=\mathbf{M}\left[\begin{array}{lll}
\mathrm{S}_{x} & \mathrm{~S}_{y} & \mathrm{~S}_{z}
\end{array}\right]^{T}=[\mathrm{MX} \mathrm{MY} \mathbf{M X}]^{T}
$$

The dynamics of the platform can be written in the following form

$$
\mathbf{A}(\mathbf{x}) \ddot{\mathbf{x}}+\mathbf{C}(\mathbf{x}, \dot{\mathbf{x}}) \dot{\mathbf{x}}+\mathbf{Q}(\mathbf{x})=-\mathbf{J}^{\mathrm{T}} \mathbf{t},
$$

where $\mathbf{A}(\mathbf{x})$ denotes the generalized inertia matrix, $\mathbf{C}(\mathbf{x}, \dot{\mathbf{x}})$ is the Coriolis and centrifugal matrix, $\mathbf{Q}(\mathbf{x})$ is a vector of gravity forces, and $\mathbf{t}$ is a vector of cable tensions. It is worth noting that the dynamics of the platform is linear in the inertial parameters i.e. in $\mathbf{I}_{p}$, MS, and $\mathbf{M}$ [22]. Let $\chi_{p} \in \mathbb{R}^{10}$ be a vector collecting the inertial parameters of the platform as

$$
\chi_{p}=[\mathrm{XX} \text { XY XZ YY YZ ZZ MX MY MZ M }]^{T} .
$$

Consequently, one can easily express the dynamics of the platform in the following regression form

$$
\Phi_{p}(\mathbf{x}, \dot{\mathbf{x}}, \ddot{\mathbf{x}}) \chi_{p}=-\mathbf{J}^{T} \mathbf{t} .
$$

The details of the regressor $\Phi_{p}(\mathbf{x}, \dot{\mathbf{x}}, \ddot{\mathbf{x}})$ is given in [15]. 


\subsection{Winches Dynamics}

The dynamics of the winches can be written as follows [15]

$$
\boldsymbol{\Gamma}_{a}=\mathbf{I}_{a} \ddot{\mathbf{q}}+\mathbf{F}_{v} \dot{\mathbf{q}}+\mathbf{F}_{c} \operatorname{sign}(\ddot{\mathbf{q}})+\mathbf{R} \mathbf{t}
$$

where $\mathbf{I}_{a} \in \mathbb{R}^{m \times m}, \mathbf{F}_{v} \in \mathbb{R}^{m \times m}$, and $\mathbf{F}_{c} \in \mathbb{R}^{m \times m}$ are diagonal matrices denoting, respectively, the inertia, the viscous friction coefficients, and the dry friction coefficients of the motors, drums and other other rotating parts. Let $\mathrm{I}_{a}, \mathrm{~F}_{v}$, and $\mathrm{F}_{c} \in \mathbb{R}^{m}$ be vectors corresponding to the diagonal elements of $\mathbf{I}_{a}, \mathbf{F}_{v}$, and $\mathbf{F}_{c}$, respectively. Then, one can gather all dynamic parameters of the winches in one vector $\chi_{a} \in \mathbb{R}^{3 m}$ defined as follows

$$
\chi_{a}=\left[\begin{array}{lll}
\mathrm{I}_{a}^{T} & \mathrm{~F}_{v}^{T} & \mathrm{~F}_{c}^{T}
\end{array}\right]^{T}
$$

Now, the winch dynamics can be written in a regression form as follows

$$
\Gamma_{a}=\Phi_{a}(\mathbf{q}, \dot{\mathbf{q}}, \ddot{\mathbf{q}}) \chi_{a}+\mathbf{R t}
$$

where $\Phi_{a}(\mathbf{q}, \dot{\mathbf{q}}, \ddot{\mathbf{q}})$ is given by

$$
\Phi_{a}(\mathbf{q}, \dot{\mathbf{q}}, \ddot{\mathbf{q}})=[\operatorname{diag}(\ddot{\mathbf{q}})|\operatorname{diag}(\dot{\mathbf{q}})| \operatorname{diag}(\operatorname{sign}(\dot{\mathbf{q}}))]
$$

As cable tensions must be within admissible non-negative ranges i.e. $0 \leq \mathbf{t}_{\text {min }} \leq$ $\mathbf{t} \leq \mathbf{t}_{\text {max }}$, the limits of the actuator torques should be within a lower and upper limits defined respectively as follows

$$
\Gamma_{a}^{\min }=\Phi_{a}(\mathbf{q}, \dot{\mathbf{q}}, \ddot{\mathbf{q}}) \chi_{a}+\mathbf{R t}_{\min }
$$

and

$$
\Gamma_{a}^{\max }=\Phi_{a}(\mathbf{q}, \dot{\mathbf{q}}, \ddot{\mathbf{q}}) \chi_{a}+\mathbf{R t}_{\max }
$$

In this paper, the dynamics of winches will be projected onto the operational space and then cable tensions will not be directly accessible. Instead, the tension distribution algorithm will be applied on the bounds defined by Eq. (19) and Eq. (20).

\subsection{CDPM Dynamics in Operational Space}

Using the first-order and second-order differential models defined by Eq. (8) and Eq. (9), one can compute the winch regression matrix in terms of either the platform or the winch acceleration, velocity, and position vectors e.g. $\Phi_{a}(\mathbf{q}, \dot{\mathbf{q}}, \ddot{\mathbf{q}}) \Leftrightarrow$ $\Phi_{a}(\mathbf{x}, \dot{\mathbf{x}}, \ddot{\mathbf{x}})$. Using Eq. (17), the cable tension can be expressed as

$$
\mathbf{t}=\mathbf{R}^{-1}\left(\boldsymbol{\Gamma}_{a}-\Phi_{a}(\mathbf{x}, \dot{\mathbf{x}}, \ddot{\mathbf{x}}) \chi_{a}\right)
$$


Substituting cable tensions in Eq. (21) into platform dynamics given by Eq. (14) yields

$$
\Phi_{p}(\mathbf{x}, \dot{\mathbf{x}}, \ddot{\mathbf{x}}) \chi_{p}-\mathbf{J}^{T} \mathbf{R}^{-1} \Phi_{a}(\mathbf{x}, \dot{\mathbf{x}}, \ddot{\mathbf{x}}) \chi_{a}=-\mathbf{J}^{T} \mathbf{R}^{-1} \boldsymbol{\Gamma}_{a}
$$

Eq. (22) can be written in a more compact form as

$$
\Phi(\mathbf{x}, \dot{\mathbf{x}}, \ddot{\mathbf{x}}) \chi=-\mathbf{J}^{T} \mathbf{R}^{-1} \Gamma_{a}
$$

where

$$
\Phi(\mathbf{x}, \dot{\mathbf{x}}, \ddot{\mathbf{x}})=\left[\Phi_{p}(\mathbf{x}, \dot{\mathbf{x}}, \ddot{\mathbf{x}})-\mathbf{J}^{T} \mathbf{R}^{-1} \Phi_{a}(\mathbf{x}, \dot{\mathbf{x}}, \ddot{\mathbf{x}})\right] \text { and } \chi=\left[\begin{array}{ll}
\chi_{p}^{T} & \chi_{a}^{T}
\end{array}\right]^{T}
$$

Solving Eq. (23) for the actuator torques $\Gamma_{a}$ taking into account the torque limits defined by Eq. (19) and Eq. (20), gives the following general solution

$$
\Gamma_{a}=\mathbf{R} \mathbf{W}^{+} \Phi(\mathbf{x}, \dot{\mathbf{x}}, \ddot{\mathbf{x}}) \chi+\mathbf{R N} \lambda,
$$

where $\mathbf{W}^{+} \in \mathbb{R}^{m \times 6}$ and $\mathbf{N} \in \mathbb{R}^{m \times m-6}$ are the Moore-Penrose pseudo-inverse and the null-space basis matrix of $-\mathbf{J}^{T}$, respectively. $\lambda \in \mathbb{R}^{m-6}$ is an arbitrary vector to be obtained such that cable tensions (and actuator torques) are admissible. For the sake of controller synthesis and analysis, the dynamic model of the CDPM expressed in terms of an inertia matrix, Coriolis, centrifugal, gravity forces, and friction forces are needed. Straightforward manipulations after substituting Eq. (15) into Eq. (12), and considering an external disturbances $\mathbf{F}_{d}$, allow us to write the dynamics of the CDPM in the following form

$$
\mathbf{A}_{e q}(\mathbf{x}) \ddot{\mathbf{x}}+\mathbf{C}_{e q}(\mathbf{x}, \dot{\mathbf{x}}) \dot{\mathbf{x}}+\mathbf{Q}_{e q}(\mathbf{x})+\mathbf{F}_{e q}(\mathbf{x}, \dot{\mathbf{x}}, \ddot{\mathbf{x}})=\mathbf{F}_{e x t}+\mathbf{F}_{d}
$$

where $\mathbf{A}_{e q}(\mathbf{x})=\mathbf{A}(\mathbf{x})-\mathbf{J}^{T} \mathbf{R}^{-1} \mathbf{I}_{a} \mathbf{R}^{-1} \mathbf{J}, \mathbf{C}_{e q}(\mathbf{x}, \dot{\mathbf{x}})=\mathbf{C}(\mathbf{x}, \dot{\mathbf{x}})-\mathbf{J}^{T} \mathbf{R}^{-1} \mathbf{I}_{a} \mathbf{R}^{-1} \dot{J}, \mathbf{Q}_{e q}(\mathbf{x})=$ $\mathbf{Q}(\mathbf{x}), \mathbf{F}_{e q}(\mathbf{x}, \dot{\mathbf{x}}, \ddot{\mathbf{x}})=-\mathbf{J}^{T} \mathbf{R}^{-1} \mathbf{F}_{v} \mathbf{R}^{-1} \mathbf{J} \dot{\mathbf{x}}-\mathbf{J}^{T} \mathbf{R}^{-1} \mathbf{F}_{c} \operatorname{sign}\left(\mathbf{R}^{-1} \mathbf{J} \dot{\mathbf{x}}\right)$, and $\mathbf{F}_{e x t}=-\mathbf{J}^{T} \mathbf{R}^{-1} \boldsymbol{\Gamma}_{a}$.

\subsection{Properties of the Dynamic Model}

Some properties of the dynamic model (26) are recalled [12]. These properties are necessary for the controller synthesis and stability analysis.

Property 1 The inertia matrix $\mathbf{A}_{e q}(\mathbf{x})$ is a positive-definite symmetric matrix and bounded away from singularity whatever the uncertainties in inertial parameters. The boundedness of $\mathbf{A}_{e q}(\mathbf{x})$ implies

$$
\lambda_{m} \mathbf{I}_{6} \leq \mathbf{A}_{e q}(\mathbf{x}) \leq \lambda_{M} \mathbf{I}_{6}
$$

or

$$
\lambda_{m} \leq\left\|\mathbf{A}_{e q}(\mathbf{x})\right\| \leq \lambda_{M}
$$

where $\mathbf{I}_{6}$ is the $6 \times 6$ identity matrix and $\lambda_{m}$ and $\lambda_{M}$ are positive scalars. 
Property 2 The external disturbance force vector $\mathbf{F}_{d}$ is assumed to be bounded i.e.

$$
\left\|\mathbf{F}_{d}\right\| \leq \zeta
$$

where $\zeta$ is a positive constant.

\section{Adaptive TSM Control of CDPM}

In this section, an adaptive TSM control is synthesized for the CDPM dynamic model given in (26). The main idea of TSM is first presented assuming that the dynamic parameters of the CDPM are known. Then, the idea is extended to the adaptive case where the dynamic parameters are assumed to be unknown and must be estimated.

\subsection{TSM Control}

For CDPM dynamics given by (26), let $\mathbf{x}_{d}, \dot{\mathbf{x}}_{d}$, and $\ddot{\mathbf{x}}_{d}$ be a desired trajectory and define $\mathbf{e}=\mathbf{x}-\mathbf{x}_{d}$ as the tracking error. The control objective is to design a feedback control law $\mathbf{F}_{\text {ext }}$ such that the platform posture $\mathbf{x}$ tracks the desired trajectory $\mathbf{x}_{d}$ in finite time.

In order to have a terminal convergence of tracking errors, a sliding surface is defined as follows [18]

$$
\mathbf{S}=\dot{\mathbf{e}}+\beta \mathbf{e}^{p / q}
$$

where $\beta>0$ is a design constant parameter, $p$ and $q$ are positive odd integers satisfying $p>q$. In order to ensure that the terminal sliding mode exists on the switching surface and equilibrium is reached in finite time, the following $\eta$-reachability condition must be satisfied [28]

$$
\frac{1}{2} \frac{\mathrm{d}}{\mathrm{dt}} \mathbf{s}^{\mathrm{T}} \mathbf{s}<-\eta|\mathbf{s}|
$$

where $\eta>0$ is a constant. If the dynamic parameters $\chi$ of the CDPM are known and the bounds on disturbance $\zeta$ are also known, then, one can choose the following control law to satisfy the $\eta$-reachability condition [19].

$$
\mathbf{F}_{e x t}=\Phi(\mathbf{x}, \dot{\mathbf{x}}, \mathbf{v}) \chi
$$

where $\mathbf{v}$ is an auxiliary control input defined by

$$
\mathbf{v}=\ddot{\mathbf{x}}_{d}+\beta \frac{q}{p} \mathbf{e}^{p / q-1} \dot{\mathbf{e}}+(\rho+\eta) \operatorname{sign}(\mathbf{s})
$$


where $\rho=\lambda_{m} \zeta$. If $\mathbf{s}(0) \neq \mathbf{0}$, then the sliding mode $\mathbf{s}=\mathbf{0}$ will be reached in finite time $t_{r}$ which satisfies $t_{r} \leq|\mathbf{s}| / \eta$. When the sliding mode is reached, the system dynamics is described by the following nonlinear differential equation

$$
\dot{\mathbf{e}}+\beta \mathbf{e}^{p / q}=0
$$

Eq. (34) has an equilibrium at $\mathrm{e}=0$ which is a globally finite-time stable attractor. And the convergence time for any initial condition $\mathrm{x}=\mathrm{x}\left(t_{r}\right)$ is finite and given by

$$
t_{s} \leq \frac{p}{\beta(p-q)}\left|\mathbf{x}\left(t_{r}\right)\right|^{1-q / p}
$$

However, the choice of such terminal sliding surface may cause singularity at convergence due to fractional power $p / q$. In addition, the control laws (32) and (33) is discontinuous and may cause chattering [19].

\subsection{Non-Singular TSM Control}

In order to avoid the singularity problem of (30), another choice of terminal sliding mode is defined as follows [20]

$$
\mathbf{s}=\mathbf{e}+\beta|\dot{\mathbf{e}}|^{\gamma} \operatorname{sign}(\dot{\mathbf{e}})
$$

where $\beta>0$ and $1<\gamma<2$.

Remark 1 . The terminal sliding mode defined by (36) has a global finite-time equilibrium i.e. for any given initial condition $\mathbf{e}(0) \neq 0$, the variable $\mathbf{e}$ reaches zero in a finite time $t_{r}$ given by [20]

$$
t_{r} \leq \frac{\gamma \beta^{1 / \gamma}}{(\gamma-1)}|\mathbf{e}(0)|^{(\gamma-1) / \gamma}
$$

Remark 2. The terminal sliding mode defined by (36) is continuous and time differentiable. Its first time derivative is written as [20]

$$
\dot{\mathbf{s}}=\dot{\mathbf{e}}+\beta \gamma|\dot{\mathbf{e}}|^{\gamma-1} \ddot{\mathbf{e}}
$$

Remark 3. Finite time stability is guaranteed for a given Lyapunov function candidate $\mathrm{V}(\mathbf{s})$ if it satisfies [20]

$$
\dot{\mathrm{V}}(\mathbf{s})+a \mathrm{~V}(\mathbf{s})+b \mathrm{~V}^{c}(\mathbf{s}) \leq 0 ; \quad a>0, b>0, \quad \text { and } 0<c<1
$$

Moreover, the finite reaching time is given by

$$
t_{r} \leq \frac{1}{a(1-c)} \ln \frac{a \mathrm{~V}^{c}(\mathbf{s}(0))+b}{b}
$$


Now, if we assume that the dynamic parameters of CDPM i.e. $\chi$ are known and the disturbance $\mathbf{F}_{d}=0$, then to design a non-singular TSM controller, let us choose the following Lyapunov function candidate

$$
\mathrm{V}=\frac{1}{2} \mathbf{s}^{\mathrm{T}} \mathbf{s}
$$

The time derivative of the non-singular TSM (38) can be rewritten as follows

$$
\dot{\mathbf{s}}=\beta \gamma|\dot{\mathbf{e}}|^{\gamma-1}\left(\beta^{-1} \gamma^{-1}|\dot{\mathbf{e}}|^{2-\gamma} \operatorname{sign}(\dot{\mathbf{e}})+\ddot{\mathbf{e}}\right)
$$

Let us choose a control law which is defined by

$$
\mathbf{F}_{e x t}=\Phi(\mathbf{x}, \dot{\mathbf{x}}, \mathbf{v}) \chi-\mathbf{K}_{1} \mathbf{s}-\mathbf{K}_{2}|\mathbf{s}|^{\rho} \operatorname{sign}(\mathbf{s})
$$

where $\mathbf{K}_{1}=\operatorname{diag}\left(k_{11}, k_{12}, \ldots k_{16}\right), \mathbf{K}_{2}=\operatorname{diag}\left(k_{21}, k_{22}, \ldots k_{26}\right)$, with $k_{i j}>0, i \in$ $\{1,2\}, j \in\{1,2, \ldots, 6\}$, and $\rho<1$, and

$$
\mathbf{v}=\ddot{\mathbf{x}}_{d}-\frac{1}{\beta \gamma}|\dot{\mathbf{e}}|^{2-\gamma} \operatorname{sign}(\dot{\mathbf{e}})
$$

The control laws (43) and (44) reduces the time-derivative of V to

$$
\dot{\mathrm{V}}=-\mathbf{s}^{T} \mathbf{H}_{1} \mathbf{s}-\mathbf{s}^{T} \mathbf{H}_{2}|\mathbf{s}|^{\rho} \operatorname{sign}(\mathbf{s})
$$

where

$$
\mathbf{H}_{1}=\operatorname{diag}\left(h_{11}, h_{12}, \ldots h_{16}\right)=\beta \gamma \lambda_{m}|\dot{\mathbf{e}}|^{\gamma-1} \mathbf{K}_{1}
$$

and

$$
\mathbf{H}_{2}=\operatorname{diag}\left(h_{21}, h_{22}, \ldots h_{26}\right)=\beta \gamma \lambda_{m}|\dot{\mathbf{e}}|^{\gamma-1} \mathbf{K}_{2}
$$

are positive diagonal matrices anywhere except at sliding mode. After straightforward manipulations of (45), one can get

$$
\dot{\mathrm{V}} \leq-2 h_{1} \mathrm{~V}-2^{(\rho+1) / 2}-h_{2} \mathrm{~V}^{(\rho+1) / 2}
$$

According to the finite-time stability (39), it is clear that (48) ensures that the tracking error e converges to zero along TSM in finite time. However, in practice, the vector of dynamic parameters $\chi$ is not exactly known since it changes with payloads. Also, external disturbances exist and must be taken into account in controller design. Therefore, an adaptive control scheme is recommended to cope with load changes and at the same time to guarantee robustness of performances. The main contribution of this paper is to extend the non-singular TSM control scheme explained above such that the dynamic parameters of CDPM are updated online via an adaptation mechanism to ensure convergence. 


\subsection{Adaptive TSM Control Scheme}

In this section, a non-singular adaptive TSM is developed for CDPM based on the classical formulations presented in Sections 3.1 and 3.2. The dynamic parameters are assumed to be unknown and have to be estimated. The main results of the proposed non-singular adaptive TSM control scheme are summarised through the following theorem.

Theorem 1. Consider a CDPM described by (23) and (26) and satisfying (27), (28), and (29) and assume that the vector of dynamic parameters $\chi$ is unknown. If the non-singular TSM (36) is chosen and the following control scheme is applied:

$$
\mathbf{F}_{\text {ext }}=\Phi(\mathbf{x}, \dot{\mathbf{x}}, \mathbf{v}) \hat{\chi}+u_{s}
$$

where $\hat{\chi}$ is an estimate of $\chi$, and

$$
\begin{aligned}
\mathrm{v} & =\ddot{\mathbf{x}}_{d}-\frac{1}{\beta \gamma}|\dot{\mathbf{e}}|^{2-\gamma} \operatorname{sign}(\dot{\mathbf{e}}), \\
u_{s} & =-\mathbf{K}_{1} \mathbf{s}-\mathbf{K}_{2}|\mathbf{s}|^{\rho} \operatorname{sign}(\mathbf{s}),
\end{aligned}
$$

and

$$
\dot{\hat{\chi}}=\beta \gamma \Lambda \Phi^{T}(\mathbf{x}, \dot{\mathbf{x}}, \mathbf{v})|\dot{\mathrm{e}}|^{1-\gamma_{\mathbf{s}}}
$$

where $\Lambda$ is a positive-definite diagonal matrix with appropriate dimensions, then the closed-loop system reaches a neighborhood of TSM in finite time and the tracking error reaches the neighborhood of the origin also in finite time.

Proof. Let $\tilde{\chi}=\chi-\hat{\chi}$ be dynamic parameters estimation error. Let us choose a Lyapunov function candidate as follows

$$
\mathrm{V}=\frac{1}{2} \mathbf{s}^{\mathrm{T}} \mathbf{s}+\frac{1}{2} \tilde{\chi}^{T} \boldsymbol{\Lambda}^{-1} \tilde{\chi}
$$

The time derivative of $\mathrm{V}$ is given by

$$
\dot{\mathrm{V}}=\mathbf{s}^{\mathrm{T}} \dot{\mathbf{s}}-\tilde{\chi}^{T} \boldsymbol{\Lambda}^{-1} \dot{\hat{\chi}}
$$

The time derivative of the terminal sliding $\mathbf{s}$ can be rewritten in the following form

$$
\dot{\mathbf{s}}=\beta \gamma|\dot{\mathbf{e}}|^{\gamma-1} \mathbf{A}_{e q}^{-1}\left(\mathbf{A}_{e q}\left(\beta^{-1} \gamma^{-1}|\dot{\mathbf{e}}|^{2-\gamma} \operatorname{sign}(\dot{\mathbf{e}})\right)+\mathbf{A}_{e q} \ddot{\mathbf{e}}\right)
$$

where $\mathbf{A}_{e q}, \mathbf{C}_{e q}, \mathbf{Q}_{e q}$, and $\mathbf{F}_{e q}$ are the inertia matrix, Coriolis and centrifugal matrix, gravity forces, and friction forces where the arguments are suppressed for simplicity. The second-order time derivative of tracking error $\ddot{\mathbf{e}}$ can be written as

$$
\mathbf{A}_{e q} \ddot{\mathbf{e}}=-\mathbf{A}_{e q} \ddot{\mathbf{x}}_{d}-\mathbf{C}_{e q} \ddot{\mathbf{x}}-\mathbf{Q}_{e q}-\mathbf{F}_{e q}+\mathbf{F}_{e x t}+\mathbf{F}_{d}
$$

Substituting (56) into (55) gives 


$$
\dot{\mathbf{s}}=\beta \gamma|\dot{\mathbf{e}}|^{\gamma-1} \mathbf{A}_{e q}^{-1}\left(\mathbf{A}_{e q}\left(\beta^{-1} \gamma^{-1}|\dot{\mathbf{e}}|^{2-\gamma} \operatorname{sign}(\dot{\mathbf{e}})-\ddot{\mathbf{x}}_{d}\right)-\mathbf{C}_{e q} \ddot{\mathbf{x}}-\mathbf{Q}_{e q}-\mathbf{F}_{e q}+\mathbf{F}_{e x t}+\mathbf{F}_{d}\right)
$$

By definition of $\mathbf{v}$ in (50), Eq. (57) can rewritten as

$$
\dot{\mathbf{s}}=\beta \gamma|\dot{\mathbf{e}}|^{\gamma-1} \mathbf{A}_{e q}^{-1}\left(-\Phi(\mathbf{x}, \dot{\mathbf{x}}, \mathbf{v}) \chi+\mathbf{F}_{e x t}+\mathbf{F}_{d}\right)
$$

Substituting the control law (51) and (49), then (58) can be simplified to

$$
\dot{\mathbf{s}}=\beta \gamma|\dot{\mathbf{e}}|^{\gamma-1} \mathbf{A}_{e q}^{-1}\left(\Phi(\mathbf{x}, \dot{\mathbf{x}}, \mathbf{v}) \tilde{\chi}-\mathbf{K}_{1} \mathbf{s}-\mathbf{K}_{2}|\mathbf{s}|^{\rho} \operatorname{sign}(\mathbf{s})+\mathbf{F}_{d}\right)
$$

Substituting (59) into $\dot{V}$ in (60) and substituting with the adaptive law (52), one can easily get

$$
\dot{\mathrm{V}} \leq \beta \gamma \mathbf{s}^{\mathrm{T}}|\dot{\mathbf{e}}|^{\gamma-1} \mathbf{A}_{e q}^{-1}\left(-\mathbf{K}_{1} \mathbf{s}-\mathbf{K}_{2}|\mathbf{s}|^{\rho} \operatorname{sign}(\mathbf{s})+\mathbf{F}_{d}\right)
$$

Using the properties (28) and (29), then one can simplify (60) as

$$
\dot{\mathrm{V}} \leq \beta \gamma \mathbf{s}^{\mathrm{T}}|\dot{\mathbf{e}}|^{\gamma-1} \lambda_{m}\left(-\mathbf{K}_{1} \mathbf{s}-\mathbf{K}_{2}|\mathbf{s}|^{\rho} \operatorname{sign}(\mathbf{s})+\zeta\right)
$$

Following the same line of proof as in [20], we can see that the system can reach neighborhood sliding-mode in finite-time which is defined by

$$
\|\mathbf{s}\| \leq \delta=\min \left(\delta_{1}, \delta_{2}\right)
$$

where $\delta_{1}=\lambda_{m} \zeta / h_{1}, \delta_{2}=\left(\lambda_{m} \zeta / h_{2}\right)^{(1 / \rho)}$. In addition, once the sliding variable is within the region $\delta$, the tracking error can reach the neighborhood of the origin also in finite time as well. This completes the proof.

\section{Simulation Study: Control of CoGiRo}

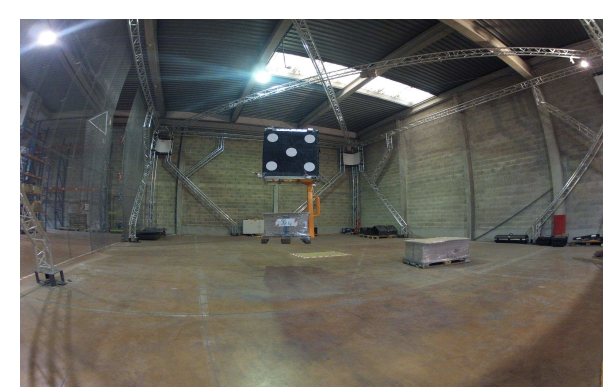

Fig. 2: CoGiRo CDPM prototype developed by LIRMM and Tecnalia 
Table 1: Cable Attach Points

\begin{tabular}{llllllll}
\hline & \multicolumn{3}{c}{ Base Points } & \multicolumn{4}{c}{ Platform Points } \\
\hline & $x_{b}$ & $y_{b}$ & $z_{b}$ & & $x_{p}$ & $y_{p}$ & $z_{p}$ \\
\hline$a_{1}$ & -7.5 & -5.5 & 6 & $b_{1}$ & 0.5 & -0.5 & 0 \\
$a_{2}$ & -7.5 & -5.5 & 6 & $b_{2}$ & -0.5 & 0.5 & 1 \\
$a_{3}$ & -7.5 & 5.5 & 6 & $b_{3}$ & -0.5 & -0.5 & 0 \\
$a_{4}$ & -7.5 & 5.5 & 6 & $b_{4}$ & 0.5 & 0.5 & 1 \\
$a_{5}$ & 7.5 & 5.5 & 6 & $b_{5}$ & -0.5 & 0.5 & 0 \\
$a_{6}$ & 7.5 & 5.5 & 6 & $b_{6}$ & 0.5 & -0.5 & 1 \\
$a_{7}$ & 7.5 & -5.5 & 6 & $b_{7}$ & 0.5 & 0.5 & 0 \\
$a_{8}$ & 7.5 & -5.5 & 6 & $b_{8}$ & -0.5 & -0.5 & 1 \\
\hline
\end{tabular}

units are in $m$

Table 2: Platform inertial parameters

\begin{tabular}{lllllllllll}
\hline Parameter & $\mathrm{XX}$ & $\mathrm{YY}$ & $\mathrm{ZZ}$ & $\mathrm{XY}$ & $\mathrm{XZ}$ & $\mathrm{YZ}$ & $\mathrm{MX}$ & $\mathrm{MY}$ & $\mathrm{MZ}$ & $\mathrm{M}$ \\
\hline$\chi_{\mathrm{PNL}}$ & 62.00 & 60.00 & 22.00 & -0.32 & -0.02 & 4.50 & 0.00 & -7.50 & 0.00 & 79.00 \\
$\chi_{\mathrm{PL}}$ & 132.00 & 135.00 & 45.00 & 0.00 & -3.00 & -2.00 & -28.00 & -7.50 & 0.00 & 200.00 \\
\hline
\end{tabular}

${ }^{a} \chi_{\mathrm{PNL}}$ and $\chi_{\mathrm{PL}}$ denote platform inertial parameters without and with load, respectively. Units are in $\mathrm{kg} . \mathrm{m}^{2}, \mathrm{~kg} . \mathrm{m}$, and $\mathrm{kg}$

Table 3: Winch dynamic parameters

\begin{tabular}{|c|c|c|c|c|}
\hline Parameter & $\mathrm{I}_{a}$ & $\mathrm{~F}_{v}$ & $\mathrm{~F}_{c}$ & $r$ \\
\hline Value & 0.0311 & 0.0200 & 3.0000 & 0.0225 \\
\hline
\end{tabular}

\subsection{CoGiRo Prototype}

The CoGiRo prototype shown in Fig. 2 is a redundantly-actuated cable-suspended CDPM developed by LIRMM and Tecnalia. CoGiRo occupies a space characterised by a $15 \mathrm{~m}$ in length, $11 \mathrm{~m}$ in width, and $15 \mathrm{~m}$ in length giving a potential workspace of $677 \mathrm{~m}^{3}$. The mobile platform is a cube with $1 \mathrm{~m}$ side length with a total mass of 79 kg. CoGiRo has 8-actuators with a 6-DOF moving platform i.e. it has 2-degrees of actuation redundancy. CoGiRo is capable of manipulating payloads of $300 \mathrm{~kg}$ over the entire workspace and up to $500 \mathrm{~kg}$ if the task to be performed is not too close to the boundaries of the workspace. The lower limit of cable tension is $\mathbf{t}_{\text {min }}=0 \mathrm{~N}$ and the upper limit is $\mathbf{t}_{\max }=5000 \mathrm{~N}$. The base and platform cable attachment points of CoGiRo which are given Table 1 in units of $m$. The inertial parameters $\chi_{\text {PNL }}$ of 
the moving platform from the CAD model of CoGiRO are given in Table 2. The inertial parameters $\chi_{\mathrm{PL}}$ of the platform with a typical payload previously used in an experimental study are used to assess the robustness of the proposed control scheme in this simulation study. The dynamic parameters of CoGiRo winches are shown in Table 3.

\subsection{Simulation Results}

Table 4: Interpolated trajectory point sequence

\begin{tabular}{lllllll}
\hline Point & $x$ & $y$ & $z$ & $\phi$ & $\theta$ & $\psi$ \\
\hline $\mathbf{x}_{0}$ & 0.000 & 0.000 & 1.296 & 0.000 & 0.000 & 0.000 \\
$\mathbf{x}_{1}$ & 0.000 & 0.000 & 2.296 & 0.000 & 0.000 & 0.000 \\
$\mathbf{x}_{2}$ & -3.850 & 1.200 & 2.296 & 0.000 & 0.000 & -45.840 \\
$\mathbf{x}_{3}$ & -3.850 & 1.200 & 1.307 & 0.000 & 0.000 & -45.840 \\
$\mathbf{x}_{4}$ & -3.000 & 2.000 & 1.307 & 0.000 & 0.000 & -45.840 \\
$\mathbf{x}_{5}$ & -3.000 & 2.000 & 1.796 & 0.000 & 0.000 & -45.840 \\
$\mathbf{x}_{6}$ & 4.000 & -1.000 & 1.796 & 0.000 & 0.000 & 11.460 \\
$\mathbf{x}_{7}$ & 4.000 & -1.000 & 1.307 & 0.000 & 0.000 & 11.460 \\
$\mathbf{x}_{8}$ & 4.300 & -2.000 & 1.307 & 0.000 & 0.000 & 11.460 \\
$\mathbf{x}_{9}$ & 4.300 & -2.000 & 2.296 & 0.000 & 0.000 & 11.460 \\
$\mathbf{x}_{10}$ & 0.000 & 0.000 & 2.296 & 0.000 & 0.000 & 0.000 \\
$\mathbf{x}_{11}$ & 0.000 & 0.000 & 1.296 & 0.000 & 0.000 & 0.000 \\
\hline
\end{tabular}

Table 5: Maximum velocity and acceleration along the trajectory

\begin{tabular}{lllllll}
\hline DOF & $x$ & $y$ & $z$ & $\phi$ & $\theta$ & $\psi$ \\
\hline Maximum velocity $\mathbf{K}_{v}[\{\mathrm{~m}, \mathrm{rad}\} / \mathrm{sec}]$ & 1.0000 & 1.0000 & 1.0000 & 1.5708 & 1.5708 & 1.5708 \\
Maximum acceleration $\mathbf{K}_{a}\left[\{\mathrm{~m}, \mathrm{rad}\} / \mathrm{sec}^{2}\right]$ & 0.1000 & 0.1000 & 0.1000 & 0.1571 & 0.1571 & 0.1571 \\
\hline
\end{tabular}

CoGiRo is supposed to perform pick-and-place tasks. In this simulation study, a trajectory of 11 mobile platform poses corresponding to a pick-and-place task is given in Table 4 . It has been generated by a 5th-order polynomial interpolation subjected to the velocity and acceleration constraints given in Table 5. In order to assess the performance of the proposed adaptive TSM control scheme, we consider two simulation cases. In Case A, the adaptive TSM controller is simulated considering only $20 \%$ of parametric uncertainty where in Case B, we consider loading the platform at configuration $\mathbf{x}_{3}$ and releasing the load at configuration $\mathbf{x}_{6}$ and, at the same 
Table 6: Adaptive TSM Controller Parameters

\begin{tabular}{cl}
\hline Parameter & Value \\
\hline$\beta$ & 1.00 \\
$\gamma$ & 1.50 \\
$\rho$ & 0.33 \\
$\mathbf{K}_{1}$ & $\operatorname{diag}\{70.00,70.00,105.00,105.00,105.00,70.00\}$ \\
$\mathbf{K}_{2}$ & $\operatorname{diag}\{25.00,25.00,37.50,25.00,25.00,30.00\}$ \\
$\Lambda_{\mathbf{I}_{p}}$ & $\operatorname{diag}\{1000.00,1000.00,1000.00,1000.00,1000.00,1000.00\}$ \\
$\Lambda_{\mathbf{M S}}$ & $\operatorname{diag}\{200.00,200.00,200.00\}$ \\
$\Lambda_{\mathbf{I}_{a}}$ & $\operatorname{diag}\{0.10,0.10,0.10,0.10,0.10,0.10,0.10,0.10\}$ \\
$\Lambda_{\mathbf{F}_{v}}$ & $\operatorname{diag}\{0.01,0.01,0.01,0.01,0.01,0.01,0.01,0.01\}$ \\
$\Lambda_{\mathbf{F}_{c}}$ & $\operatorname{diag}\{0.10,0.10,0.10,0.10,0.10,0.10,0.10,0.10\}$ \\
\hline
\end{tabular}

time, the adaptive TSM control algorithm is initialised with $20 \%$ of parametric uncertainty for the no-load platform inertial parameters $\chi_{P N L}$. The parameters shown in Table 6 have been used in simulation of both cases where $\Lambda_{i}$ is the adaptation gains corresponding to the parameters of $i$.

Fig. 3, 4, 5, and 6 show the simulation results of Case A. It can be seen that the proposed adaptive TSM control scheme is able to achieve good tracking performance even with parametric uncertainty. Fig. 7, 8, 9, and 10 show the simulation results of Case $B$. It can be seen that at the loading instant, both cable-tension and control input torques increase instantaneously. However the adaptive TSM is able to restore the tracking error in short period of time and achieves a good tracking performance over the whole trajectory.

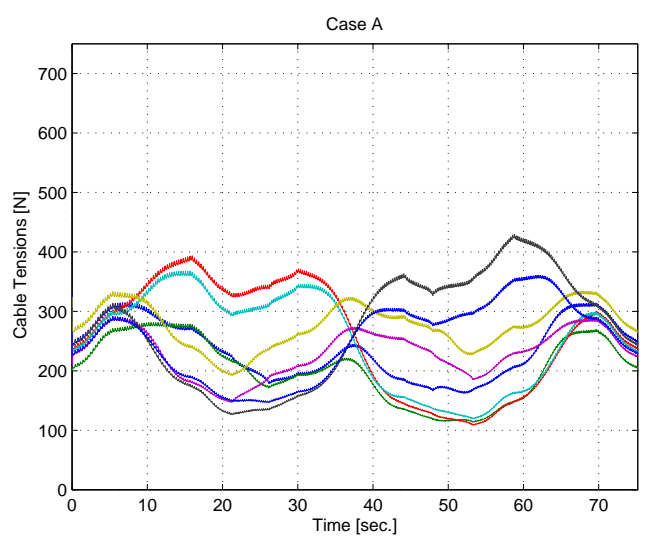

Fig. 3: Cable Tensions: Case (A) 


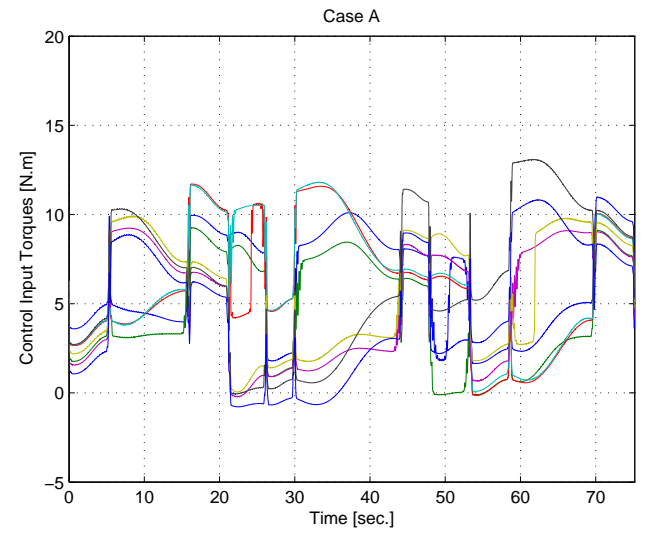

Fig. 4: Control Input Torques: Case (A)

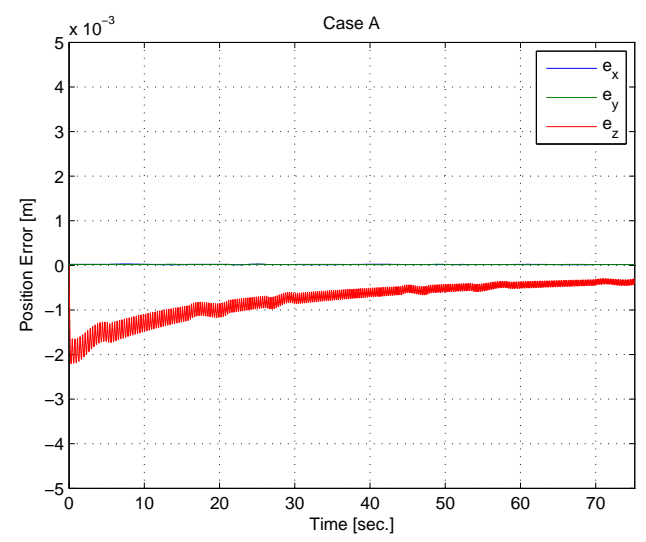

Fig. 5: Position Error: Case (A)

\section{Conclusion}

The main contribution of this paper is extending an early developed non-singular TSM control by synthesizing an adaptive law for CDPM dynamic parameters. The main motivation of using TSM for CDPMs is its finite-time stability which generally results in high tracking performances. Stability of conventional TSM for robotic manipulators are based on the assumption that the dynamics are partially known while the uncertain part is bounded. This assumption is true, however, it may results in large control input torques especially when heavy payloads are to be manipulated. To tackle this problem, an adaptive mechanism is introduced to estimate online the dynamic parameters. The overall dynamics of CDPM has been reformulated in this paper in a regression form to facilitate the design of the adaptive control. Redun- 


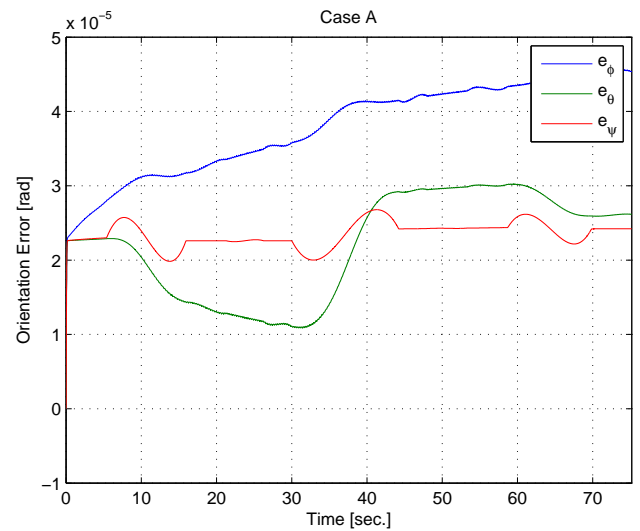

Fig. 6: Orientation Error: Case (A)

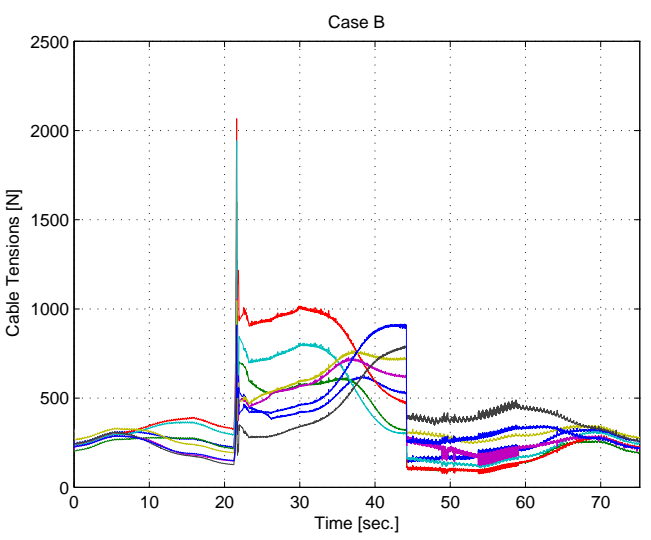

Fig. 7: Cable Tensions: Case (B)

dancy resolution is then solved based on the limits of control input torques corresponding to the tension limits. The adaptive TSM control scheme has been applied in simulation to a large cable-suspended CDPM - CoGiRo. CoGiRo is supposed to perform pick-and-place of heavy payloads. The simulations show the effectiveness of the proposed scheme for large values of parametric uncertainties and loading conditions. However, the real benefit of the proposed adaptive TSM control will appear through experimental studies on CoGiRo. Our future work will be dedicated to these experimental validations. 


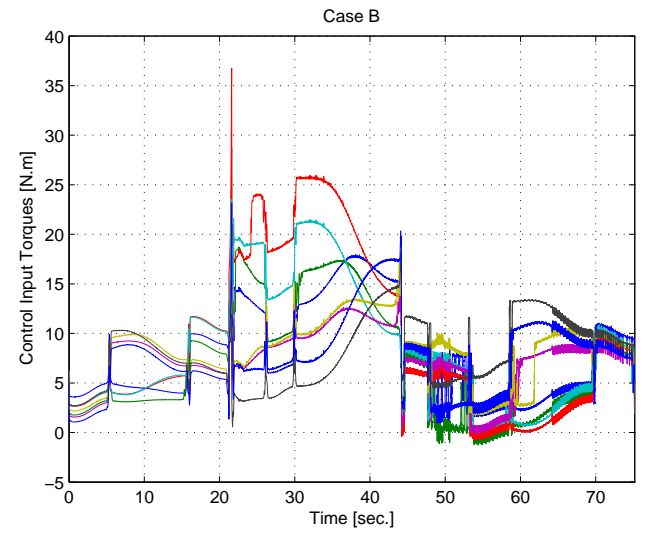

Fig. 8: Control Input Torques: Case (B)

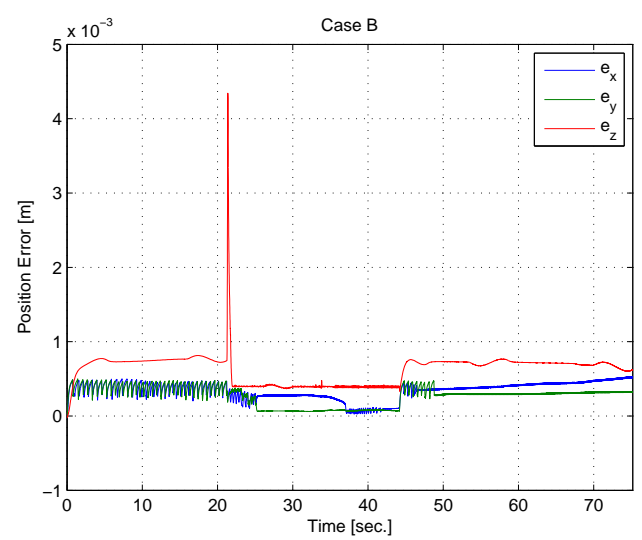

Fig. 9: Position Error: Case (B)

\section{References}

[1] Kawamura, S., Kino, H., Won, C.: High-speed manipulation by using parallel wire-driven robots. Robotica, 18, pp. 13-21 (2000).

[2] Shiang, W-J., Cannon, D., Gorman, J.: Dynamic Analysis of Cable Attay Robotic Crane. In Proc. IEEE International Conference on Robotics and Automation, ICRA1999 pp. 2495-2500, Detroit, Michigan, USA (1999).

[3] Lamaury, J.: Contribution à la commande des robots parallèles à câble à redondance d'actionnement. Ph.D. thesis, Université Montpellier II, 2013.

[4] Lamaury, J., Gouttefarde, M., Tempier, O.: Design and Control Strategies of a Redundant Suspended Cable-Driven Parallel Robot. Latest Advances in Robot Kinematics, ARK2012, pp. 1-9, Innsbruck, Austria (2012). 


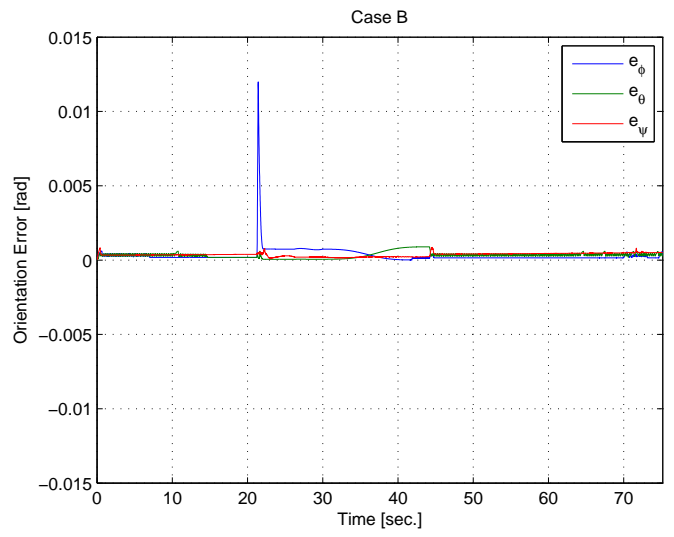

Fig. 10: Orientation Error: Case (B)

[5] Lamaury, Lamaury, J., Gouttefarde, M.: A Tension Distribution Method with Improved Computational Efficiency. In Proc. First International Conference on Cable-Driven Parallel Robots, pp. 71-85, Stuttgart, Germany (2012).

[6] Fang, S., Franitza, D., Torlo, M., Bekes, F., Hiller, M.: Motion control of a tendon-based parallel manipulator using optimal tension distribution. IEEE/ASME Transactions on Mechatronics, 9, pp. 561-568 (2004).

[7] Bruckmann, T., Pott, A., Hiller, M., Franitza, D.: A Modular Controller for Redundantly Actuated Tendon-Based Stewart Platforms. EuCoMes, The First Conference on Mechanism Science, Obergurgl, Austria (2006).

[8] Oh, S-R., Agrawal, S.K.: A reference governor-based controller for a cable robot under input constraints. IEEE Transactions on Control Systems Technology, 13, pp. 639-645 (2005).

[9] Pott, A., Bruckmann, T., Mikelsons, L.: Closed-form Force Distribution for Parallel Wire Robots. In Proc. 5th International Workshop on Computational Kinematics, pp. 25-34 (2009).

[10] Mikelsons, L., Bruckmann, T., Hiller, M., Schramm, D.: A real-time capable force calculation algorithm for redundant tendon-based parallel manipulators. IEEE International Conference on Robotics and Automation, ICRA2008, pp. 3869-3874 (2008).

[11] Gholami, P., Aref, M., Taghirad, H. D.: On The Control of the KNTU CDRPM: A Cable Driven Redundant Parallel Manipulator. IEEE International Conference on Intelligent Robots and Systems, IROS2008, pp. 24042409 (2008).

[12] Khosravi, M.A., Taghirad, H.D.: Robust $\{$ PID $\}$ control of fully-constrained cable driven parallel robots. Mechatronics, 24(2), pp. 87-97 (2014).

[13] Kino, H., Yahiro, T., Fumiaki, T., Morizono, T.: Robust PD Control Using Adaptative Compensation for Completely Restrained Parallel-Wire Driven Robots. IEEE Transactions on Robotics, 23(4), pp. 803-812 (2007). 
[14] Williams II, R.L., Gallina, P., Vadia, J.: Planar Translational Cable-DirectDriven Robots. Journal of Robotic Systems, 20(3), pp. 107-120 (2003).

[15] Lamaury, J., Gouttefarde, M., Chemori, A., Herve, P.E.: Dual-space adaptive control of redundantly actuated cable-driven parallel robots. International Conference on Intelligent Robots and Systems (IROS), 2013 IEEE/RSJ, pp. 4879-4886 (2013).

[16] Wijesoma, S. W., Richard, R.J.: Robust trajectory following of robots using computed torque structure with VSS. International Journal of Control, 52(4), pp. 935-962 (1990).

[17] Oh, S-R., Agrawal, S. K., Nonlinear Sliding Mode Control and Feasible Workspace Analysis for a Cable Suspended Robot with Input Constraints and Disturbances. In Proc. American Control Conference, pp. 4631-4636, Boston, MA, USA (2004).

[18] Man, Z., Paplinski, A.P., Wu, H.R.: A robust MIMO terminal sliding mode control scheme for rigid robotic manipulators. IEEE Transactions on Automatic Control, 39(12), pp. 2464-2469 (1994).

[19] Feng, Y., Yu, X., Man, Z.: Non-singular terminal sliding mode control of rigid manipulators. Automatica, 38(12), pp. 2159-2167 (2002).

[20] Yu, S., Yu, X., Shirinzadeh, B., Man, Z.: Continuous finite-time control for robotic manipulators with terminal sliding mode. Automatica, 41(11), pp. 1957-1964 (2005).

[21] Feng, Y., Yu, X., Han, F.: On nonsingular terminal sliding-mode control of nonlinear systems. Automatica, 49(6), pp. 1715-1722 (2013).

[22] Khalil, W., Dombre, E.: Modeling, Identification and Control of Robots. Elsevier Science (2004).

[23] Mondal, S., Mahanta, C.: Adaptive second order terminal sliding mode controller for robotic manipulators. Journal of the Franklin Institute, 351(4), pp. 2356-2377 (2014).

[24] Bruckmann, T., Mikelsons, L., Brandt, T., Hiller, M., Schramm, D.: Wire Robots Part I: Kinematics , Analysis \& Design. In Parallel Manipulators New Developments, pp. 109-132 (2008).

[25] Bruckmann, T., Mikelsons, L., Brandt, T., Hiller, M., Schramm, D.: Wire Robots Part II: Dynamics, Control \& Application. In Parallel Manipulators New Developments, pp. 133-152 (2008).

[26] Diao, X., Ma, Ou.: Vibration analysis of cable-driven parallel manipulators. Multibody System Dynamics, 21, pp. 347-360 (2009).

[27] Craig, J.: Adaptive Control of Merchanical Manipulators. Addison-Wesley (1988).

[28] Slotine, J-J.E., Li, W.: Applied Nonlinear Control. Prentice Hall (1991). 